\title{
Rotación de Directivos Públicos. ¿Problema de gestión o solución de confianza política?
}

\section{Rotation of Public Managers. Problem of management or solution of political trust?}

INFORMACIÓN DEL

\section{ARTÍCULO}

Fecha de recepción: 31 de Octubre de 2018.

Fecha de aceptación: 25 de Abril de 2019.

${ }^{1} \mathrm{PhD}$ en Administración, Universidad Andina Simón Bolívar. Docente e investigadora del Instituto de Altos Estudios Nacionales-Ecuador.

E-mail: irma.jara@iaen.edu.ec

Código ORCID:

https://orcid.org/0000-0002-6342-4068

${ }^{2}$ Magíster en Relaciones Internacionales, Universidad Central del Ecuador Becaria del Programa de Doctorado en Estudios Internacionales en la Facultad Latinoamericana de Ciencias Sociales, FLACSO.

E-mail: jcedenio2000@yahoo.es

Código ORCID:

https://orcid.org/0000-0002-1990-5438

CITACIÓN: Jara Íñiguez, I., \& Cedeño Alcívar, J. (2019). Rotación de Directivos Públicos. ¿Problema de gestión o solución de confianza política? Podium, 35, 1-22. doi:10.31095/podium.2019.35.1

ENLACE DOI:

http://dx.doi.org/10.31095/podium.201 9.35 .1

Irma Jara Íñiguez ${ }^{1}$, Jenny Cedeño Alcívar ${ }^{2}$

\section{Resumen}

El estudio analiza la rotación de los directivos públicos de alto nivel en Ecuador y su utilización como instrumento de confianza política desde el Ejecutivo, en contraste con un modelo de ciclo de vida del desempeño directivo, para determinar si es un problema para la obtención de resultados en la gestión. Se realiza un análisis cualitativo y diacrónico de los índices de rotación y el tiempo de permanencia en el cargo de los directivos desde una muestra de Ministerios que representan un peso importante con relación al modelo de gestión de Estado para el quinquenio 2012-2016; como resultado, se muestra que con altos niveles de rotación se configuran problemas para efectivizar una dirección profesional $\mathrm{y}$, aunque esta sea utilizada como herramienta política y electoral para el movimiento de las autoridades, a dichos niveles disminuye su utilidad estratégica.

\section{Palabras Clave:}

Administración Pública, Ciclo de vida, Confianza, Directivos Públicos, Ecuador, Rotación.

Clasificación JEL: 83, D73, J63.

\begin{abstract}
The study analyzes the rotation of high level public managers in Ecuador and their use as an instrument of political trust from the Executive, in contrast to a life cycle model of managerial performance, to determine if it is a problem to obtain results in the management. A qualitative and diachronic analysis of rotation rates and the time of permanence in the position of the managers is carried out from a sample of Ministries that represent an important weight in relation to the State management model for the five-year period 2012-2016; as a result, it is shown that with high levels of turnover problems are configured to effect a professional management and, although this is used as a political and electoral tool for the movement of the authorities, at these levels its strategic utility decreases.

Keywords:

Public Administration, Life Cycle, Confidence, Public Managers, Ecuador, Rotation.
\end{abstract}

JEL Classification: H83, D73, J63. 


\section{Introducción}

La administración pública ecuatoriana presenta un entorno complejo para la gestión de las autoridades y directivos públicos, como responsables de las políticas públicas del Gobierno y de los resultados obtenidos (Ramió y Alsina, 2016); ya que por una parte, están los problemas derivados de las características de las sociedades contemporáneas que se trasladan a los gobiernos y sus organizaciones (Longo, 2009), que obligan a diseñar intervenciones $\mathrm{y}$ reformas que empujan a los directivos a asumir enfoques basados en el ensayo y la rectificación; y, por otra, en el marco de la nueva gestión pública, se requiere que los funcionarios tengan múltiples habilidades técnicas y políticas, capacidad de generar seguridad $y$ confianza a la ciudadanía y al mandatario en el ejercicio del poder.

En este tipo de escenarios complejos, la implementación de estrategias, acciones y el desempeño de una gestión eficaz requiere de períodos largos para su maduración y ejecución (Longo, 2009), lo que conlleva una lógica de permanencia referencial en un cargo, a fin de desarrollar y superar las fases o etapas en el ejercicio del puesto de directivo, a lo largo del tiempo. Pero en un sistema presidencialista, la alta rotación de autoridades públicas da cuenta, por un lado, de la amplia discrecionalidad de los Presidentes para estructurar o reestructurar el Gobierno, como ha sucedido en el Ecuador donde en promedio, estos cambiaron el $21,3 \%$ de su gabinete por año entre 1979 y 1996, el 19,4\% entre
1996 y 2007 y el 23,2\% a partir de 2007 (Polga-Hecimovich, Basabe, y Mejía, 2012); $\mathrm{y}$, por otro, de la falta de interés de los nuevos dignatarios y autoridades por establecer continuidad en las acciones, a través de directivos estables, que vayan más allá de la ejecución de un periodo de gobierno y que aseguren la consecución de políticas de Estado y objetivos de largo plazo.

Conforme afirman Boyne, James, John y Petrovsky (2010 a) las autoridades suponen que los burócratas que comparten la misión del político de turno necesitan menos control del día a día y, por lo tanto, se les confía la ejecución del trabajo a quienes tienen la misma línea política; por otro lado, si los directivos sienten que no son aceptados o si no comparten la misma inclinación política que el nuevo gobernante preparan su salida. Consecuentemente, las organizaciones públicas se ven afectadas por la corta permanencia de los directivos derivada de la brevedad de los ciclos político -electorales, como circunstancia inherente al sector público. Sin embargo, la rotación de directivos públicos también ocurre en períodos largos de estabilidad política, debido a la discrecionalidad como potestad presidencial, ya que, como sostiene Martínez-Gallardo (2012) una autoridad presidencial fuerte se puede asociar con mayor inestabilidad del gabinete, al tener menos incentivos para comprometerse y, más aún si no existe un contrapeso en el legislativo o no se tiene necesidad del consenso. A su vez, la rotación y los cambios ministeriales son herramientas de ajuste que permiten: hacer frente a externalidades, optimizar el apoyo político, reordenar las fuerzas 
políticas, mejorar las competencias del gabinete, $\mathrm{y}$, mejorar la imagen del gobierno (González-Bustamante y Olivares, 2016; Camerlo y Pérez-Liñán, 2015; Martínez- Gallardo, 2012).

Existen escasos estudios de rotación en el sector público latinoamericano que cubran el nivel jerárquico superior y la alta dirección pública, no sólo en primera, sino segunda y tercera línea gubernamental. Entre los estudios de gabinete y ministeriales se cuentan los de: González-Bustamante y Olivares (2015; 2016) y, Avendaño y Dávila (2012) sobre Chile; Camerlo y Pérez-Liñán (2015) sobre Argentina; Franz y Codato (2018) sobre Brasil; y, Camerlo y Martínez-Gallardo (2018) y, Martínez -Gallardo $(2011 ; 2014)$ respecto a varios países en América, sin embargo, no abarcan análisis específicos sobre rotación en Ecuador en cuanto a directivos a nivel de ministros, viceministros y subsecretarios, salvo los datos de volatilidad a nivel ministerial anotados por Basabe-Serrano, Polga-Hecimovich y Mejía (2017) y, Polga-Hecimovich et al (2012). El entorno complejo en el que se desempeñan los directivos públicos, los niveles de rotación y cortos periodos de permanencia en el cargo evidencian la importancia de la realización de la investigación en cuanto al caso Ecuador que permita responder la pregunta al respecto de si la rotación laboral es un problema para la obtención de resultados en la gestión o una herramienta útil de confianza política, en el marco de una investigación más amplia que se encuentra en desarrollo.
Para responder la pregunta, se realiza un estudio diacrónico comparado para el periodo 2012-2016, con análisis cualitativo de la función ejecutiva en Ecuador, contrastando los resultados periódicos -semestral- de la rotación de ministros, viceministros y subsecretarios de Estado y su permanencia en el cargo, con las tendencias posibles dentro del ciclo de vida de la gestión del directivo público. Se utilizan como referentes las proposiciones formuladas al modelo de ciclo de vida directivo aplicado al sector público por Ysa y Salvador (2015), y la teoría del ciclo de vida de un directivo que propone una relación como $\mathrm{U}$ invertida entre el ejercicio del directivo y el desempeño (Wulf et al, 2010).

Como casos de estudio se seleccionaron 8 carteras de Estado, correspondientes a los sectoriales de Finanzas, Asuntos Internos, Defensa, Exterior, Salud, Educación, Inclusión Social, y Electricidad, a partir de los criterios de su peso respecto a la ejecución presupuestaria, la importancia en el discurso con relación al modelo de gestión de Estado y, de su gestión en el gabinete para la toma de decisiones, así como, la distribución de ministerios por tipo de prestigio realizados por Krook y O Brien (2012).

Los resultados muestran los altos niveles de rotación de directivos públicos alcanzados por sectorial y en promedio $y$, tiempos de permanencia en el cargo con un máximo de 2,6 años y un mínimo de 1,3 años. Con estos datos, tomando como referencia el esquema y fases del ciclo de 
vida para la gestión directiva pública, no se viabilizaría el desarrollo de las etapas con rendimiento creciente $y$ no se aseguraría un proceso no perjudicial en cuanto a la gestión o como estrategia política.

\section{Revisión de literatura}

\section{Dirección Pública y ciclo de vida}

El concepto de ciclo de vida del directivo, entendido como una serie de etapas desde el ingreso hasta la desvinculación de la institución, permite analizar los cambios o evolución de la gestión del directivo durante el ejercicio de su cargo que tienen impacto en los resultados de las organizaciones (Ysa y Salvador, 2015) y, por lo tanto, es fundamental el análisis en contraste con el tiempo de permanencia del directivo, debido a la rotación.

Ysa y Salvador (2015) aplicaron al sector público el modelo de ciclo de vida directivo, seminal de Hambrick y Fukutomi (1991), y elaboraron sobre su clasificación de 5 etapas, una propuesta de proposiciones adaptada al sector, las que se utilizarán para este estudio. Estas 5 etapas o fases discernibles dentro del cargo o posición de dirección, a su vez dan lugar a distintos patrones y se componen de dimensiones que son consideradas para medir los cambios y son: el compromiso con un paradigma; nivel de conocimiento de las tareas; diversidad de la información a la que se accede; interés en las tareas; $y$, poder.

Las cinco etapas del ciclo de vida directivo aplicado al sector público por Ysa y Salvador (2015) y sus proposiciones, en resumen, son:

(a) respuesta al mandato, proposición 1: se dedica primordialmente a responder el mandato de quien lo designó, hasta que goce de su soporte político. Bajo conocimiento y alto interés en las tareas, obtención de información de fuentes diversas y bajo poder. Se tiende a desarrollar con un periodo estimado de duración de 1 a 2 años.

(b) experimentación, proposición 2: el directivo luego de relajar su compromiso con sus paradigmas de inicio intenta nuevos enfoques para dirigir la entidad $y$, trata de probar otros métodos, con un periodo estimado de duración de 1 a 2 años.

(c) selección de una narrativa, proposición 3: el directivo tiende a seleccionar un esquema sobre cómo se debe configurar y gestionar la organización, se compromete a reformular el paradigma; aumenta la capacidad para un mejor desempeño, con un periodo estimado de duración de 1 a 2 años.

(d) convergencia, proposición 4: las acciones implementadas sirven para converger hacia el esquema definido, y completa la curva de aprendizaje. Amplio conocimiento, disminuye interés en las tareas, obtención de información de pocas fuentes filtradas y amplio poder. Periodo estimado de duración de 3 a 5 años.

(e) disfunción, proposición 5: ya no 
existe aprendizaje y el interés por la tarea es mínimo, la eficiencia o crecimiento del rendimiento disminuye o se estanca. $\mathrm{Su}$ principal incentivo es mantener el poder. Su duración corresponde al resto de años de permanencia, luego de las anteriores fases.

Con este esquema, en promedio, los directivos cuya duración abarca las cinco etapas (lo que dure la etapa 5, más 6 o 11 años, según el mínimo o máximo) experimentarán su máximo rendimiento en algún punto intermedio en su tiempo en el cargo, y teniendo en cuenta que la teoría institucional del ciclo de vida directivo acorde a lo indicado por Wulf et al (2010) propone una relación de $U$ invertida entre el mandato y el desempeño del directivo, se desprende que el desempeño muy temprano y muy tarde en el ejercicio será menor.

En este contexto al analizar la gestión del directivo público, si se parte de este modelo y enfoque teórico enunciado, es necesario prestar atención a los periodos de permanencia en el puesto para que el directivo pueda desempeñarse y evitar que su muy corta o larga permanencia en el puesto pueda operar como una limitación para la obtención de resultados $\mathrm{y}$, por lo tanto, a la dirección profesional.

De la designación de los directivos públicos por confianza política y la rotación

El directivo público político, es aquel que, en relación con un principio de legitimidad democrática, ocupa un puesto de responsabilidad en la Administración pública, encontrándose entre sus funciones la de impulsar, en su ámbito sectorial de actuación, la implementación de las políticas públicas del Gobierno, ser interlocutor de la institución o unidad que dirige, representarla, dirigir el trabajo de los subordinados y ser responsable de los resultados obtenidos (Ramió y Alsina, 2016).

En la administración pública, los cargos políticos se refieren a aquellos cuyo ingreso no es a través de pruebas y entrevistas objetivas en las que se evalúe el mérito y la capacidad de los candidatos, sino que su designación se fundamenta básicamente en la confianza política. La legitimidad electoral es la condición necesaria y suficiente para investir al político de su función, la cual es traspasada indirectamente a los representantes designados para la administración (Alcántara, 2013).

En relación con la rotación de los directivos públicos, en un estudio realizado en 18 países latinoamericanos desde 1980 al 2003 entre los que consta Ecuador, Escobar-Lemmon y Taylor-Robinson (2009) evidencian que en promedio los ministros se mantienen en el cargo 2,17 años y el $46 \%$ se mantienen en el cargo un año. Carteras como Finanzas y Relaciones Exteriores tienden a tener una menor rotación, mientras que Interior, Justicia, Trabajo y Seguridad Social tienen un nivel de rotación bastante mayor, las carteras del sectorial social se sitúan en un punto intermedio (Escobar-Lemmon y Taylor-Robinson, 2010 en Franco Mayorga, 2014). 
La rotación de los directivos públicos y su tiempo de permanencia en el puesto es un problema cuando estos no pueden desarrollar y completar las etapas de su ciclo de vida ascendente; y, durante el corto tiempo en el ejercicio, se encuentran avocados a dar respuestas rápidas $\mathrm{y}$ visibles en relación con el discurso del mandante, lo que se corresponde con la etapa 1 del esquema de ciclo de vida propuesto. Adicionalmente, desde un enfoque de costo-beneficio, la literatura indica que la relación entre la rotación y el rendimiento organizativo es la de una curva en forma de U invertida, por lo que, la rotación en niveles bajos a moderados generalmente benefician el desempeño organizativo, ya que al poder ingresar personal más capacitado o con experiencias distintas esto es una oportunidad favorable para el aprendizaje, pero tales beneficios disminuyen a medida que aumenta el porcentaje de rotación, ya que se imponen más costos que beneficios (Lee, 2017; Ryu y Lee, 2013; Hur, 2013; Park y Shaw, 2013).

Si bien la gestión de la rotación -a una movilidad razonable- puede facilitar la incorporación de nuevos puntos de vista y ampliar la experiencia del personal, por otro lado, la pérdida de un funcionario valioso es disfuncional ya que implica disminución de la eficacia general de la organización. Por consiguiente, si el efecto de la rotación depende del desempeño (funcionario y organización) $\mathrm{y}$, también de los costos asociados, ya que, los costos -económicos y políticosde la retención pueden ser mayores que los costos de rotación, sin importar cuán alto sea el desempeño; las autoridades a partir de la comprensión de la afectación al desempeño de la organización deben tomar acciones para abordar el problema (Hur, 2013; Boyne, James, John, y Petrovsky, 2011).

Pero, en la administración de la rotación, incluso si no existe un problema de desempeño como causa de los resultados obtenidos, mover a un directivo público puede ser una forma de que los políticos convenzan a la ciudadanía de que fueron estos los responsables de los resultados inadecuados. Sin embargo, la frecuencia en que los políticos pueden cambiar de administrador también puede verse limitada por la necesidad de atraer y retener directivos competentes (Boyne et al, 2010 a).

\section{Caso Ecuador}

Sistema presidencial y puestos de dirección política y administrativa

El sistema político ecuatoriano tiene un régimen presidencialista, en el que el control sobre la administración pública se ejerce a través de la capacidad presidencial de nominación y remoción política de su gabinete, sin necesidad de que los apruebe o vete la Función Legislativa.

La Ley Orgánica de Servicio Público, LOSEP (2010) define su artículo 83, literal a) a quienes ocupan puestos de dirección política y administrativa del Estado, los que a su vez están excluidos de la carrera en el servicio público, entre los que se encuentran los ministros, 
viceministros y subsecretarios de Estado. Estos cargos se encuentran tipificados como de libre nombramiento y remoción y el artículo 85 de la LOSEP estipula que las autoridades nominadoras podrán designarlos, previo el cumplimiento de los requisitos previstos para el ingreso al servicio público, y removerlos libremente.

Los Ministros de Estado son competentes para el despacho de todos los asuntos inherentes a sus ministerios sin necesidad de autorización alguna del presidente de la República, salvo los casos expresamente señalados en leyes especiales, por otro lado, el número y atribuciones de los subsecretarios de cada Ministerio, es definido por el respectivo Ministro, acorde con lo estipulado en el Estatuto de Régimen Jurídico Administrativo de la Función Ejecutiva (Decreto Ejecutivo 2428, 2002).

\section{Rotación y Permanencia de miembros de Gabinete Presidencial}

Desde hace décadas, en Ecuador, los constantes cambios realizados por los jefes de Estado han dado lugar a una alta tasa de rotación de ministros, evidenciándose esto como un problema, al ser un obstáculo para la ejecución y continuidad de las políticas públicas. Esta rotación ministerial se incrementó sustancialmente en la década 1997-2007 ya que los presidentes no lograron terminar su período, como fue el caso de Abdalá Bucaram, Jamil Mahuad y Lucio Gutiérrez. Sin embargo, de forma posterior al 2007, con la elección del presidente Rafael Correa, quien ejerció como tal hasta el 2017, la rotación se mantuvo alta a pesar de que gobernó en un clima de relativa estabilidad, con importantes niveles de apoyo popular y mayoría legislativa en la Asamblea Nacional (2010), atribuyéndose esto a su propia estrategia presidencial (Basabe-Serrano et al 2017; Freidenberg y Pachano, 2016).

Según datos de Polga-Hecimovich et al (2012) en Freidenberg y Pachano (2016), en promedio, los presidentes cambiaron el 19,4\% de su gabinete entre 1996 y 2007 y el 23,2\% a partir de 2007. Entre 1997 y 2007 la mayoría de los ministros ejerció el cargo menos de doce meses. Entre 2007 y 2017 los ministros tuvieron un promedio menor a dos años en el cargo, siendo, en la administración de Correa buena parte de los cambios una rotación interna entre los miembros del gabinete (PDBA, 2011; CEGE, 2017).

A partir de 2007 y hasta mayo de 2017 los gabinetes fueron de un mismo partido o afinidad política y las pautas para la designación y sucesión de ministros han respondido más a criterios de orden ideológico y de coincidencia con el proyecto de la denominada revolución ciudadana (Basabe-Serrano et al, 2017; Freidenberg y Pachano, 2016; Polga-Hecimovich, 2013), es decir, en términos de confianza política; $y$, adicionalmente acorde con el discurso se han incluido criterios de género $\mathrm{y}$ generacionales.

\section{Metodología}

Se estudia como caso la función ejecutiva en Ecuador por el periodo 
2012-2016, y se exploran y describen aspectos de rotación de los directivos públicos-políticos con alcance a los niveles del jerárquico superior correspondiente a ministros, viceministros y subsecretarios, a fin de indagar problemas e identificar variables de gestión relacionados con el tiempo de permanencia, en contraste con las fases del modelo del ciclo del desempeño en el cargo; por lo que, el estudio tiene un alcance exploratorio y componentes descriptivos acorde con la definición de Hernández-Sampieri, Fernández y Baptista (2014).

Se realizó un análisis cualitativo a partir de una investigación documental de 2012-2016, complementada con información bibliográfica y de hemeroteca. Se analizó también la información de carácter cuantitativa derivada de la gestión de talento humano, en específico lo relacionado con la planificación y movimientos del nivel directivo evidenciados desde el distributivo mensual del sector, buscando generar datos descriptivos para la discusión respecto al significado desde la perspectiva conceptual y de gestión.

Se trabajó con un esquema basado en un estudio diacrónico del marco político electoral, informes de rendición de cuentas, acciones relacionadas con la designación del nivel directivo de la función ejecutiva en Ecuador; $\mathrm{y}$, contrastando los resultados periódicos -semestrales- de la rotación con la viabilidad y coherencia de las acciones posibles dentro del esquema del ciclo de vida directivo adaptado al sector público en la propuesta de Ysa y Salvador (2015) -utilizado como referencia teórica y académica-, descrito en el marco teórico y según Figura 1, y triangulando el análisis de los datos obtenidos con otras perspectivas y bajo diferentes visiones, con el propósito de responder si los niveles de rotación de la muestra de gabinete presidencial seleccionado se revelan como herramienta de utilidad para asegurar la confianza política, en detrimento de posibilitar una gestión pública profesional.

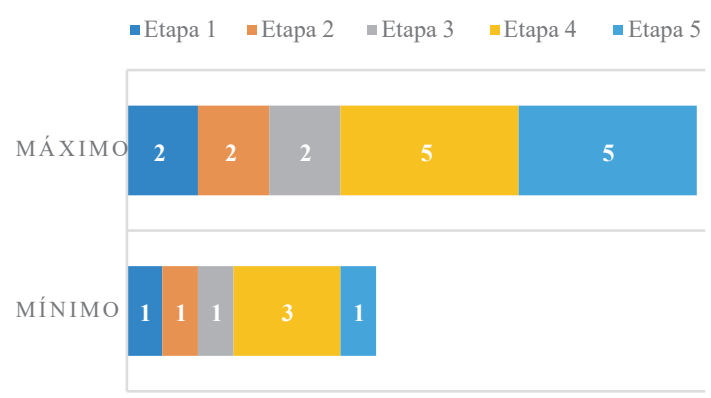

Figura 1. Duración por Etapas del Ciclo de Vida para el Directivo Público (años).

Fuente: Ysa y Salvador (2015) adaptado de Hambrick y Fukutomi (1991). Elaboración: Propia.

En el estudio del caso, para la recolección de datos, se tomó como muestra -no estadística- a casos tipo que compartan las características para el análisis (Hernández-Sampieri, Fernández y Baptista, 2014), en términos de relevancia y prestigio en el ámbito de los gabinetes presidenciales conformados desde 2012 hasta 2016. El estudio comprendió como nivel directivo público a los funcionarios del jerárquico superior de nivel de ministros, viceministros y subsecretarios. Se tomó como muestra a 8 ministerios seleccionados a partir de un análisis de su peso respecto a la ejecución presupuestaria, la importancia en el discurso con relación al modelo de 
gestión de Estado y, de su gestión en el gabinete para la toma de decisiones.

Para determinación del peso por la importancia en el discurso, se tomó como referencia el Plan Nacional de Desarrollo 2013-2017, en el que se establece en su objetivo 2: auspiciar la igualdad, la cohesión y la equidad social y territorial en la diversidad; en el objetivo 3: mejorar la calidad de vida de la población; en el objetivo 4: fortalecer las capacidades y potencialidades de la ciudadanía (SENPLADES, 2013). Identificándose de éstos la importancia de los sectoriales de educación, salud e inclusión.

En el mismo instrumento, el objetivo 6 que se enfoca en fortalecer la seguridad integral y el objetivo 12 dirigido a garantizar la soberanía y la paz, profundizar la inserción estratégica en el mundo y la integración latinoamericana, revelan la importancia de los sectoriales de asuntos internos, defensa nacional y asuntos del exterior. Los objetivos 7, $10 \mathrm{y}$ 11 relacionados con promover la sostenibilidad ambiental, la transformación de la matriz productiva y la eficiencia de los sectores estratégicos, evidencian la importancia del sectorial de relacionado con energía (SENPLADES, 2013).

Se utilizaron complementariamente, otorgando más rigor a la muestra utilizada, los criterios que categorizan a los ministerios por el tipo de prestigio en términos de su visibilidad y control significativo sobre la política acorde con la tipificación presentada por Krook y O Brien (2012). Las carteras relacionadas con los sectoriales de finanzas, asuntos internos, defensa, exterior y energía ${ }^{4}$ son de alto prestigio en esta clasificación; mientras que las educación, salud y bienestar social son de medio prestigio.

La selección de Ministerios en este contexto y con los criterios anotados, incluyó a los de sectores que representan un peso importante y amplio en el gabinete presidencial, sector social, estratégicos, económico, seguridad y conocimiento y talento humano, en concordancia además con las políticas públicas y objetivos del Plan Nacional de Desarrollo. Los Ministerios seleccionados para el estudio fueron: a) Finanzas; b) Interior; c) Defensa; d) Relaciones Exteriores; e) Educación; f) Salud; d) Inclusión Económica y Social; y e) Electricidad y Energía Renovable. La Tabla 1 resume los aspectos considerados para la selección de los 8 Ministerios Sectoriales del estudio.

Tabla 1.

Aspectos considerados para selección de Ministerios

\begin{tabular}{lllll}
\hline Sectorial & $\begin{array}{l}\text { Presupuesto } \\
\text { \% Devengado } \\
\text { Sectorial }\end{array}$ & $\begin{array}{l}\text { Discurso/ } \\
\text { PND }\end{array}$ & $\begin{array}{l}\text { Peso } \\
\text { Gabinete }\end{array}$ & $\begin{array}{l}\text { Tipo } \\
\text { Prestigio }\end{array}$ \\
$\begin{array}{l}\text { Finanzas/ Tesoro } \\
\text { Asuntos Internos }\end{array}$ & $50,80^{5}$ & Alto & Alto & Alto \\
$\begin{array}{l}\text { Defensa Nacional } \\
\text { Asuntos del }\end{array}$ & 5 & Alto & Alto & Alto \\
$\begin{array}{l}\text { Exterior } \\
\text { Educación }\end{array}$ & 0,47 & Alto & Alto & Alto \\
$\begin{array}{l}\text { Salud } \\
\text { Bienestar Social }\end{array}$ & 12,8 & Alto & Alto & Alto \\
Recursos Naturales 4,28 & Alto & Alto & Medio \\
\hline
\end{tabular}

Fuente: Ministerio de Finanzas (2017); SENPLADES (2013); Krook y O Brien (2012). Elaboración propia.

Para el análisis de la rotación y permanencia se recolectaron los datos teniendo como fuente los distributivos

\footnotetext{
${ }^{4}$ Acorde a la clasificación realizada por Krook y O Brien (2012), para los miembros de la OPEP, cualquier ministerio que tenga que ver con recursos naturales, petróleo o energía se considera de alto prestigio.

${ }^{5}$ Incluye Participación del presupuesto codificado del sectorial tipificado con Tesoro Nacional
} 
oficiales de las instituciones de la Función Ejecutiva proporcionados por el Ente rector en materia laboral (MDT, 2017), se realizó la revisión y depuración para homogenizar los datos por tipo, clasificarlos y filtrarlos en los niveles requeridos para el estudio y se realizó la validación de datos contrastando con las fuentes institucionales (MDN; MEF; MINEDUC; MIES; MDI; MSP; Cancillería; MEER, 2018). Se realizaron cortes semestrales dentro del periodo 2012-2016 y se consideraron los datos del inicio y el final de dichos cortes, para la determinación de las salidas de funcionarios por entidad y por periodo, la rotación y el periodo de permanencia resultante. Para confirmación y correspondencia se contrastaron los datos cuantitativos con los cualitativos determinados desde el análisis documental, lo que permitió determinar consistencia y validez del estudio.

Luego de un proceso de corroboración estructural mediante el cual se verificó que las categorías y tipos de datos se soportaban mutuamente, la rotación del equipo directivo de los Ministerios para el estudio, se midió conforme la fórmula:

$$
\mathrm{Rd}=(\mathrm{Vd}+\mathrm{Dd} / 2) \times 100 /(\mathrm{F} 1+\mathrm{F} 2) / 2
$$

donde:

$\mathrm{Rd}=$ Porcentaje de rotación de directivos públicos: ministros, viceministros y subsecretarios de Estado.

$\mathrm{Vd}=$ Vinculaciones en la institución de funcionarios del equipo directivo.

$\mathrm{Dd}=$ Desvinculaciones de la institución de funcionarios del equipo directivo.

F1= Número de funcionarios del equipo directivo al inicio del periodo en la institución.

F2= Número de funcionarios del equipo directivo al final del periodo en la institución.

\section{Resultados}

Los resultados evidencian que durante el periodo de 2012-2016 se efectuó un alto nivel de movimiento del equipo de autoridades designadas a ejercer la dirección política del Estado. Como se desprende de las Figuras 2 y 3, las carteras de defensa e inclusión son las que presentan más salidas de ministros con 4 y 3 cambios en 5 años, representando esto que los ministros permanecieron en el cargo entre 1 y 1,3 años; en educación, salud y finanzas los ministros permanecieron en promedio 2,5 años y únicamente en las carteras relaciones exteriores e interior los ministros se mantuvieron más de 6 años (2010-2016) y 5 años $(2011-2016)^{6}$ respectivamente, cubriendo en el periodo de estudio, la mayoría de este.

Camerlo y Pérez- Liñán (2015) indican que la evidencia empírica confirma que la supervivencia del ministro es considerablemente más frágil durante los tiempos difíciles, pero también muestra que los líderes pueden tomar decisiones selectivas sobre la retención del ministro cuando aseguran su popularidad. Esto puede evidenciarse para el caso del ministerio del interior, en el que conforme se evidencia en la Figura 2, el ministro se mantuvo en el cargo en el periodo de análisis y su salida en el 2016 responde a su candidatura por participación en las elecciones encabezando la lista para asambleístas por el partido de Gobierno Alianza País (González, 2016), en este

${ }^{6}$ Para estos dos casos en que los ministros permanecieron desde etapa anterior al periodo de estudio, se consideró este particular. 
periodo el Presidente Correa mantuvo una popularidad superior entre el $58 \%$ en el 2012 y el 52\% en el 2016 (CEDATOS, 2019); y, en el caso de relaciones exteriores, la explicación se relaciona con el hecho de asegurar el poder y preferencias del Ejecutivo conforme su estrategia (Basabe-Serrano et al 2017; Abad, 2016; Polga-Hecimovich, 2013), dada su popularidad y aceptación aún frente a las críticas por la colocación del ministro.

Los periodos en los que se efectúo el mayor número de movimientos de las más altas autoridades sectoriales fueron el primer semestre de 2012 y el último semestre de 2016, ambos coinciden en ser periodos previos a eventos electorales presidenciales y legislativos para nuevos periodos de gobierno en 2013 y 2017; por lo que, lo cambios corresponden a estrategias pre candidaturas y también debido a movimientos de autoridades para apoyo en campaña y por participación como candidatos (ver Figuras 2 y 3 ).

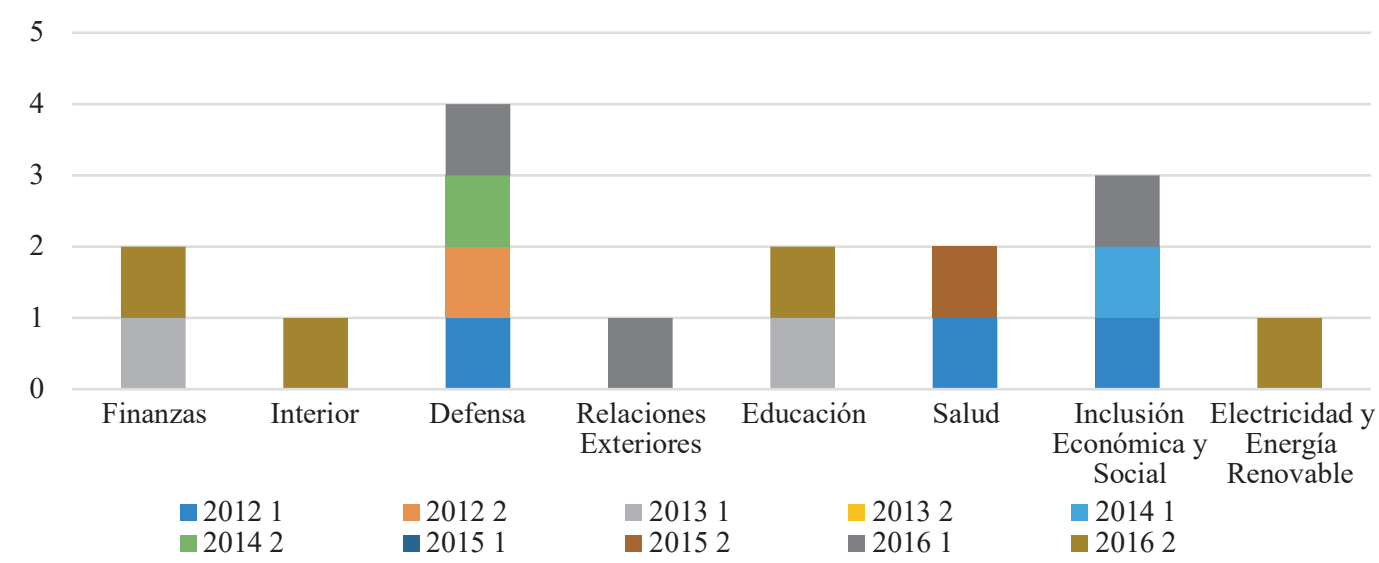

Figura 2. Salida semestral de ministros sectoriales.

Fuente: CEGE (2017); Ministerio del Trabajo (2017). Elaboración propia.

5

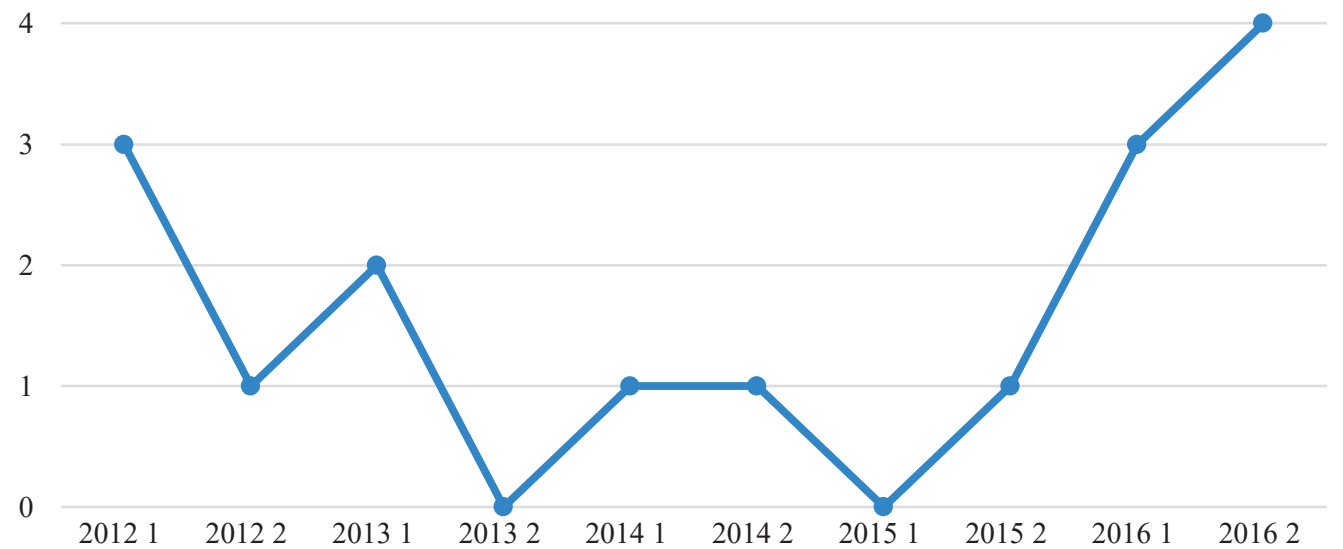

Figura 3. Salida semestral de ministros. Total, Ministerios Estudio.

Fuente: CEGE (2017); Ministerio del Trabajo (2017). Elaboración propia. 
Los cambios en época previa electoral, son concordantes, como argumento en la administración pública, con lo indicado por Boyne et al (2010a) referido a que el control de la burocracia por parte de los políticos para lograr sus objetivos es clásico $\mathrm{y}$, que los gobernantes usan una gama de controles, en este caso de forma previa a las elecciones, para ajustar el aparato administrativo de forma que les permita traducir sus expectativas o necesidades políticas en actividades y resultados así como mejorar sus expectativas electorales (Polga -Hecimovich, 2013). Si bien, las expectativas para las elecciones del periodo 2013, eran favorables para el Gobierno de Correa, está claro que al 2012 existe un fin de ciclo, acompañado de acciones para rendir cuentas y mostrar los avances luego de un periodo completo, que hacía ya tiempo no se acostumbraba en el País. A su vez, anticiparse a conformar equipos para la implementación de un nuevo plan de desarrollo, así como nuevas metas que alcanzar.

En relación a los cambios realizados en el 2016, anticipados a las elecciones 2017, en este periodo a diferencia de las elecciones del 2013, existiría un cambio de mandante ya sea por el mismo partido o por un partido diferente, por lo que en esta coyuntura, las autoridades designadas por Correa como titular al 2016, no necesariamente compartirían los valores o preferencias de la nueva administración; en este caso, los cambios respondían básicamente - a excepción de los casos de renuncias efectivas - : a) remplazos debido a que los titulares debían responder a participación en otras curules políticas; b) anticiparse a conformar equipos que puedan responder mejor a los ajustes requeridos para las elecciones; $y, c)$ dar respuesta en relación al desempeño de tipo sanción (Polga-Hecimovich, 2013; Boyne, James, John, y Petrovsky, 2010b).

$\mathrm{Al}$ analizar a los equipos directivos de viceministros y subsecretarios de Estado, las carteras que más movimiento y salidas presentan son las de educación, inclusión y relaciones exteriores, con 32, 31 y 24 salidas de funcionarios respectivamente, de un total de 11 y 10 puestos del equipo directivo.

Los periodos en los que se efectuó el mayor número de movimientos del equipo ministerial fueron el primer semestre de 2012 y el penúltimo semestre de 2016, ambos coinciden con cambios importantes de las autoridades ministeriales por lo que, al tiempo que se cambian ministros procede un cambio de los equipos y, dependiendo de las nuevas autoridades estos recambios o refrescamiento suelen ser inmediatos o al cabo de uno máximo dos meses (Ver Figuras 4 y 5).

Este refrescamiento es una forma de asegurar la implementación de las preferencias respecto a la respuesta al mandato del ministro electo y para ello se acude a remover a los funcionarios que pueden haber estado estrechamente asociados con la autoridad anterior, y designar a nuevos directivos o personal del jerárquico superior; pero asimismo, el poder para reemplazar al equipo, como agentes de la máxima autoridad, es una fuente importante de influencia a 
demostrar (Boyne et al, 2010 a; 2010 b).

Si bien se verifica que los cambios de los equipos de directivos, de manera general coinciden en el caso con los cambios de ministros lo que se sostiene en el argumento establecido de la confianza y lealtad política conforme lo indicado por Boyne et al (2010 a; 2010 b), no obstante, cuando los equipos cambian, incluso sin una nueva administración, como es el caso de las carteras de educación, inclusión social y relaciones exteriores, los políticos en ejercicio tienen otro incentivo gracias a las doctrinas establecidas en la argumentación administrativa para la nueva gestión pública, como el de preocuparse por el desempeño inadecuado o malos resultados de los directivos, debido a su incidencia en procesos electorales en el futuro

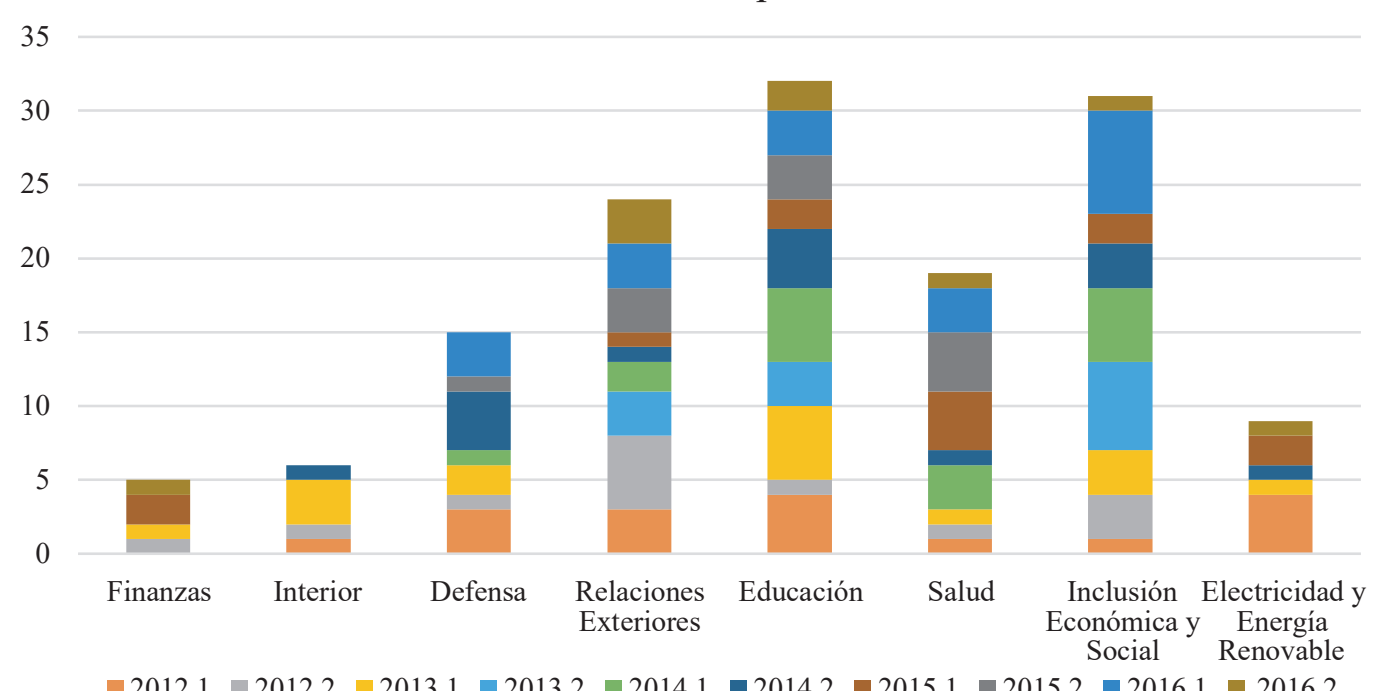

Figura 4. Salida semestral de viceministros y subsecretarios de Estado, Sectorial.

Fuente: Ministerio del Trabajo (2017). Elaboración propia.

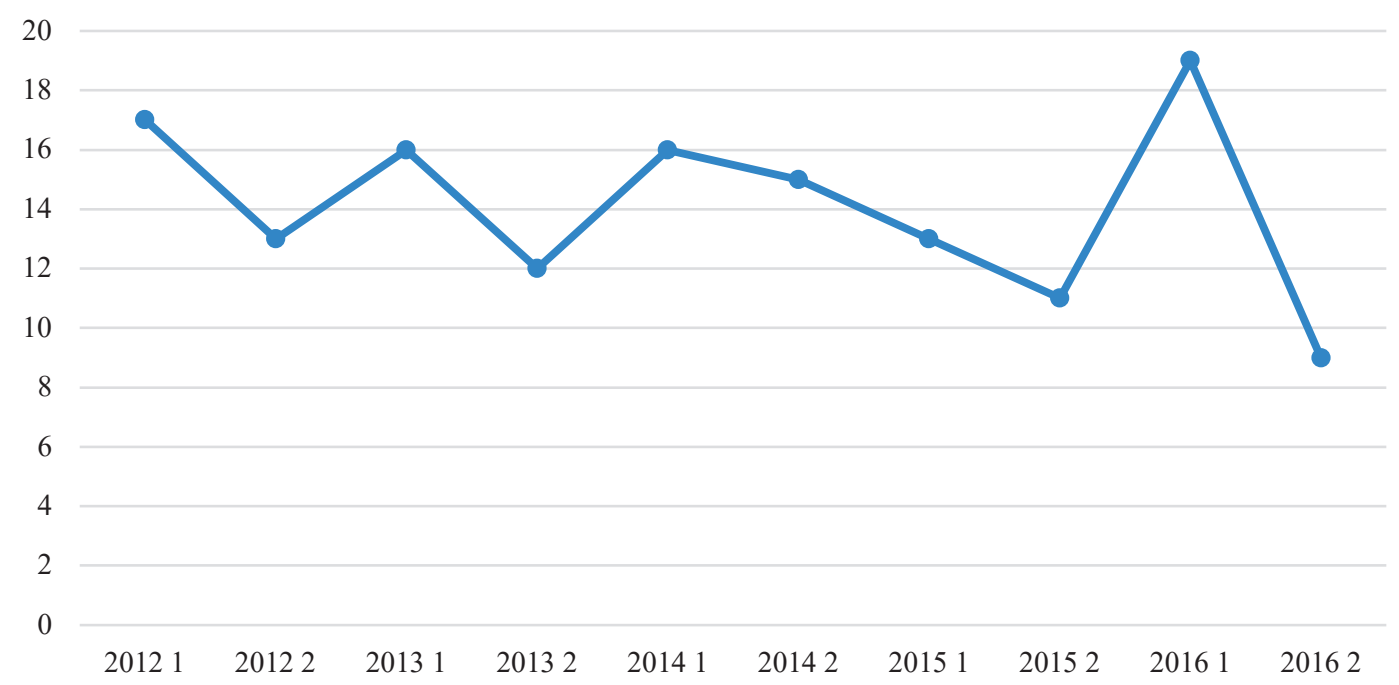

Figura 5. Salida semestral de viceministros y subsecretarios de Estado. Total, Ministerios Estudio.

Fuente: Ministerio del Trabajo (2017). Elaboración propia. 
Tabla 2.

Salida semestral de ministros, viceministros y subsecretarios de Estado

\begin{tabular}{lccccccccccc}
\hline & $\mathbf{2 0 1 2}$ & $\mathbf{2 0 1 2}$ & $\mathbf{2 0 1 3}$ & $\mathbf{2 0 1 3}$ & $\mathbf{2 0 1 4}$ & $\mathbf{2 0 1 4}$ & $\mathbf{2 0 1 5}$ & $\mathbf{2 0 1 5}$ & $\mathbf{2 0 1 6}$ & $\mathbf{2 0 1 6}$ & $\mathbf{T}$ \\
Finanzas & $\mathbf{1}$ & $\mathbf{2}$ & $\mathbf{1}$ & $\mathbf{2}$ & $\mathbf{1}$ & $\mathbf{2}$ & $\mathbf{1}$ & $\mathbf{2}$ & $\mathbf{1}$ & $\mathbf{2}$ & \\
Interior & 1 & 1 & 2 & 0 & 0 & 0 & 2 & 0 & 0 & 2 & 7 \\
Defensa & 4 & 2 & 3 & 0 & 0 & 1 & 0 & 0 & 0 & 1 & 7 \\
R. Exterior & 3 & 5 & 0 & 3 & 2 & 1 & 1 & 3 & 4 & 3 & 25 \\
Educación & 4 & 1 & 6 & 3 & 5 & 4 & 2 & 3 & 3 & 3 & 34 \\
Salud & 2 & 1 & 1 & 0 & 3 & 1 & 4 & 5 & 3 & 1 & 21 \\
Inclusión & 2 & 3 & 3 & 6 & 6 & 3 & 2 & 0 & 8 & 1 & 34 \\
Electricidad & 4 & 0 & 1 & 0 & 0 & 1 & 2 & 0 & 0 & 2 & 10 \\
Total & 20 & 14 & 18 & 12 & 17 & 16 & 13 & 12 & 22 & 13 & 157 \\
\hline
\end{tabular}

Fuente: Ministerio del Trabajo (2017). Elaboración propia.

(Basabe-Serrano et al 2017; Abad, 2016; Polga-Hecimovich, 2013).

La Tabla 2 muestra, que en un periodo de 5 años se movieron de sus puestos a 157 funcionarios de alto nivel jerárquico superior a cargo de la dirección política del Estado en sectoriales de gran prestigio por el monto de recursos asignados, su inclusión en el discurso político y su peso en el gabinete ligado a la toma de decisiones. Estas salidas o cambios arrojan a su vez, un porcentaje de rotación promedio del $29 \%$ en total. Evidenciándose la mayor rotación promedio en las carteras de inclusión, defensa y salud, con 51\%, 43\% y 39\% respectivamente; y la mayor rotación promedio por periodo en el primer semestre de 2012 y 2016, determinándose a su vez que en estos semestres las carteras de salud y defensa realizaron movimientos de vinculación y desvinculación abarcando en número al $100 \%$ de sus funcionarios de equipo, es decir renovándolo, Tabla 3.

Con estos niveles de movimientos y rotación de directivos públicos la Tabla 4 muestra el número de directivos públicos que han ejercicio durante el periodo de estudio y la permanencia promedio en el cargo, para ministros, viceministros $\mathrm{y}$ subsecretarios de Estado, en los que se evidencia una permanencia máxima de 2,6 años y mínima de 1,3 años, lo que

Tabla 3.

Rotación semestral de directivos públicos (ministros, viceministros y subsecretarios de Estado)

\begin{tabular}{lccccccccccc}
\hline & $\mathbf{2 0 1 2}$ & $\mathbf{2 0 1 2}$ & $\mathbf{2 0 1 3}$ & $\mathbf{2 0 1 3}$ & $\mathbf{2 0 1 4}$ & $\mathbf{2 0 1 4}$ & $\mathbf{2 0 1 5}$ & $\mathbf{2 0 1 5}$ & $\mathbf{2 0 1 6}$ & $\mathbf{2 0 1 6}$ & $\begin{array}{c}\text { Rd } \\
\text { Prom }\end{array}$ \\
Finanzas & $\mathbf{1}$ & $\mathbf{2}$ & $\mathbf{1}$ & $\mathbf{2}$ & $\mathbf{1}$ & $\mathbf{2}$ & $\mathbf{1}$ & $\mathbf{2}$ & $\mathbf{1}$ & $\mathbf{2}$ & $7 \%$ \\
Interior & $6 \%$ & $6 \%$ & $18 \%$ & $0 \%$ & $6 \%$ & $0 \%$ & $18 \%$ & $0 \%$ & $0 \%$ & $18 \%$ & $7 \%$ \\
Defensa & $9 \%$ & $8 \%$ & $30 \%$ & $17 \%$ & $0 \%$ & $33 \%$ & $0 \%$ & $0 \%$ & $0 \%$ & $20 \%$ & $12 \%$ \\
R. Exterior & $20 \%$ & $71 \%$ & $56 \%$ & $0 \%$ & $20 \%$ & $90 \%$ & $11 \%$ & $11 \%$ & $100 \%$ & $0 \%$ & $43 \%$ \\
Educación & $50 \%$ & $11 \%$ & $0 \%$ & $42 \%$ & $46 \%$ & $25 \%$ & $16 \%$ & $26 \%$ & $47 \%$ & $25 \%$ & $29 \%$ \\
Salud & $100 \%$ & $20 \%$ & $25 \%$ & $42 \%$ & $50 \%$ & $30 \%$ & $29 \%$ & $29 \%$ & $21 \%$ & $29 \%$ & $35 \%$ \\
Inclusión & $71 \%$ & $63 \%$ & $85 \%$ & $59 \%$ & $69 \%$ & $33 \%$ & $22 \%$ & $0 \%$ & $88 \%$ & $18 \%$ & $51 \%$ \\
Electricidad & $50 \%$ & $0 \%$ & $8 \%$ & $9 \%$ & $0 \%$ & $17 \%$ & $27 \%$ & $0 \%$ & $9 \%$ & $9 \%$ & $13 \%$ \\
Total & $47 \%$ & $28 \%$ & $34 \%$ & $22 \%$ & $30 \%$ & $30 \%$ & $20 \%$ & $15 \%$ & $41 \%$ & $17 \%$ & $29 \%$ \\
\hline
\end{tabular}

Fuente: Ministerio del Trabajo (2017). Elaboración propia. 
contrastado con las etapas del ciclo de vida del directivo, en atención al esquema de duración mínima del ciclo de vida propuesto por Hambrick y Fukutomi (1991) y adaptado por Ysa y Salvador (2015), coloca el ejercicio del cargo en la etapa 2, que corresponde a la de experimentación para el caso de todas las carteras, a excepción de la de finanzas en la que el desempeño llega hasta la etapa 3 que corresponde a la de selección de discurso, Figura 6. La Figura 7,

Tabla 4.

Número y Permanencia Promedio en el Puesto de Directivos Públicos (Autoridades) ${ }^{7}$.

\begin{tabular}{lccccc}
\hline Ministerio & Ministros & Viceministros & Subsecretarios & $\begin{array}{c}\text { Permanencia } \\
\text { Promedio } \\
\text { (meses) }\end{array}$ & $\begin{array}{c}\text { Permanencia } \\
\text { Promedio } \\
\text { (años) }\end{array}$ \\
Finanzas & 2 & 4 & 11 & 32 & 2,6 \\
Interior & 18 & 4 & 5 & 24 & 2,0 \\
Defensa & 5 & 3 & 15 & 16 & 1,3 \\
R. Exterior & 2 & 9 & 24 & 19 & 1,6 \\
Educación & 2 & 7 & 33 & 16 & 1,3 \\
Salud & 3 & 8 & 18 & 17 & 1,4 \\
Inclusión & 4 & 10 & 27 & 15 & 1,2 \\
Electricidad & 2 & 1 & 12 & 24 & 2,0 \\
\hline
\end{tabular}

Fuente: Ministerio del Trabajo (2017). Elaboración propia.

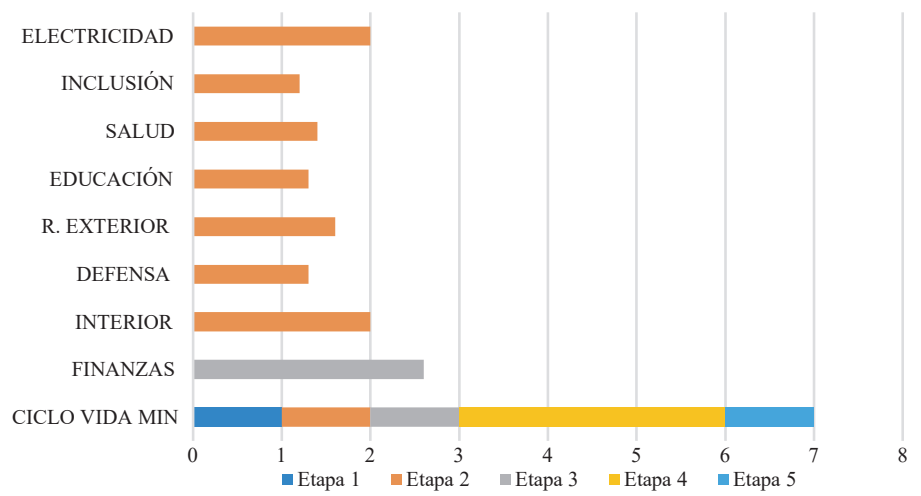

Figura 6. Etapas de Ciclo de Vida de Directivo Público Sectorial. Promedio 2012-2016. Ciclo de vida mínimo Fuente: Elaboración propia.

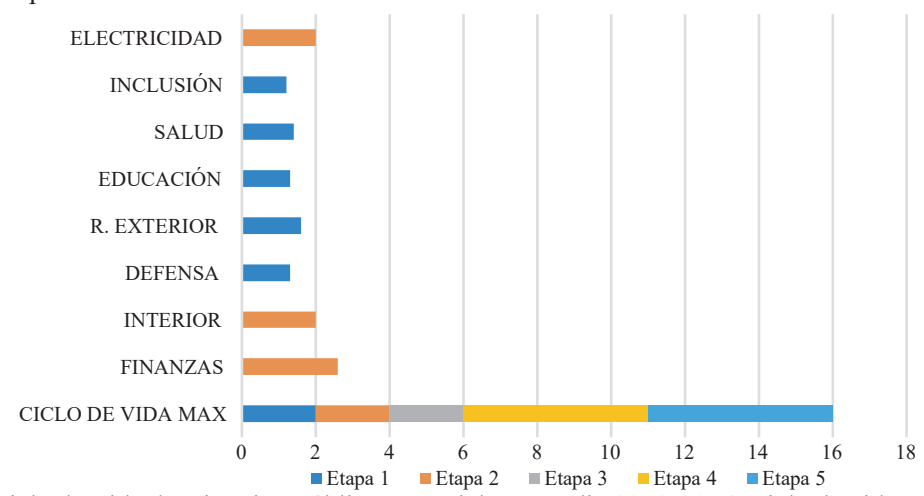

Figura 7. Etapas de Ciclo de Vida de Directivo Público Sectorial. Promedio 2012-2016. Ciclo de vida máximo. Fuente: Elaboración propia.

${ }^{7}$ En los casos en que la estructura variaba se realizó el cálculo con el número de puestos a diciembre de 2016. El promedio se realiza teniendo en cuenta todos los tipos de puestos seleccionados, ministros, viceministros y subsecretarios de Estado.

${ }^{8}$ A la salida del ministro José Serrano, se nombró un encargado, dentro del periodo de estudio. 
relacionada con el ciclo de vida en atención al esquema de duración máxima, utilizada como referencia para este estudio, coloca el ejercicio de los directivos de las carteras de inclusión, salud, educación, exterior y defensa en la etapa 1 y a las carteras de electricidad, finanzas e interior, en la etapa 2.

En el análisis de ambos esquemas de duración del ciclo de vida, únicamente para el caso de la cartera de Finanzas se ubica en la etapa 3 , en el caso de duración mínima de etapas, lo cual implica que únicamente en este caso el aspecto relacionado con el conocimiento de

Tabla 5 .

Tendencias en el Ciclo de Vida del Directivo Público Sectorial 2012-2016. Escenario 1.

\begin{tabular}{|c|c|c|c|c|c|}
\hline & $\begin{array}{l}\text { Compromiso con } \\
\text { un paradigma }\end{array}$ & $\begin{array}{l}\text { Conocimiento de } \\
\text { tareas }\end{array}$ & $\begin{array}{l}\text { Diversidad de } \\
\text { información }\end{array}$ & $\begin{array}{l}\text { Interés } \\
\text { en la tarea }\end{array}$ & Poder \\
\hline $\begin{array}{l}\text { Etapa } 2 \\
\text { Interior } \\
\text { Defensa } \\
\text { R. Exterior } \\
\text { Educación } \\
\text { Salud } \\
\text { Inclusión } \\
\text { Electricidad }\end{array}$ & $\begin{array}{l}\text { Puede ser fuerte o } \\
\text { débil }\end{array}$ & $\begin{array}{l}\text { Moderado; algo } \\
\text { creciente }\end{array}$ & $\begin{array}{l}\text { Muchas fuentes, } \\
\text { pero cada vez más } \\
\text { filtradas }\end{array}$ & Alto & $\begin{array}{l}\text { Moderado; } \\
\text { creciendo }\end{array}$ \\
\hline $\begin{array}{l}\text { Etapa } 3 \\
\text { Finanzas }\end{array}$ & $\begin{array}{l}\text { Moderadamente } \\
\text { fuerte }\end{array}$ & $\begin{array}{l}\text { Alto; ligeramente } \\
\text { creciente }\end{array}$ & $\begin{array}{l}\text { Pocas fuentes; } \\
\text { moderadamente } \\
\text { filtradas }\end{array}$ & $\begin{array}{l}\text { Moderadamente } \\
\text { alto }\end{array}$ & $\begin{array}{l}\text { Moderado; } \\
\text { creciendo }\end{array}$ \\
\hline
\end{tabular}

Fuente: Ysa y Salvador (2015) adaptado de Hambrick y Fukutomi (1991); Ministerio del Trabajo (2017). Elaboración propia.

Tabla 6.

Tendencias en el Ciclo de Vida del Directivo Público Sectorial 2012-2016. Escenario 2.

\begin{tabular}{|c|c|c|c|c|c|}
\hline & $\begin{array}{l}\text { Compromiso con } \\
\text { un paradigma }\end{array}$ & $\begin{array}{l}\text { Conocimiento de } \\
\text { tareas }\end{array}$ & $\begin{array}{l}\text { Diversidad de } \\
\text { información }\end{array}$ & $\begin{array}{l}\text { Interés en la } \\
\text { tarea }\end{array}$ & Poder \\
\hline $\begin{array}{l}\text { Etapa } 1 \\
\text { Defensa } \\
\text { R. Exterior } \\
\text { Educación } \\
\text { Salud } \\
\text { Inclusión }\end{array}$ & $\begin{array}{l}\text { Moderadamente } \\
\text { fuerte }\end{array}$ & $\begin{array}{l}\text { Bajo pero } \\
\text { creciendo } \\
\text { rápidamente }\end{array}$ & $\begin{array}{l}\text { Muchas fuentes; } \\
\text { sin filtrar }\end{array}$ & Alto & $\begin{array}{l}\text { Bajo; } \\
\text { creciendo }\end{array}$ \\
\hline $\begin{array}{l}\text { Etapa } 2 \\
\text { Finanzas } \\
\text { Interior } \\
\text { Electricidad }\end{array}$ & $\begin{array}{l}\text { Puede ser fuerte } \\
\text { o débil }\end{array}$ & $\begin{array}{l}\text { Moderado; algo } \\
\text { creciente }\end{array}$ & $\begin{array}{l}\text { Muchas fuentes, } \\
\text { pero cada vez } \\
\text { más filtradas }\end{array}$ & Alto & $\begin{array}{l}\text { Moderado; } \\
\text { creciendo }\end{array}$ \\
\hline
\end{tabular}

Fuente: Hambrick y Fukutomi (1991); Ministerio del Trabajo (2017). Elaboración propia. 
cambios en el equipo de alta dirección conducen a mejoras cuando el desempeño previo es malo, pero conducen al deterioro cuando el desempeño previo es bueno, y que el efecto de este tipo rotación es asimétrico, es decir que el perjuicio provocado por el cambio en las organizaciones de alto desempeño superaría los beneficios para las organizaciones de bajo rendimiento; esto lleva a cuestionar la decisión de un cambio político cuando el desempeño organizativo es adecuado.

Por lo que, cuando las medidas adoptadas como estrategias políticas no son causadas por el bajo desempeño o resultado inadecuado en las políticas y, motivadas con el efecto sanción, es posible que esto afecte la dirección profesional del nuevo directivo y el desempeño de la organización. Independientemente de esto, es decir si se excluye el factor de buen o mal desempeño organizativo previo, es importante atender el tránsito en el ciclo de vida, ya que el resultado dependerá también de la etapa en que se encuentre su ejercicio como directivo público (Ysa y Salvador, 2015).

En todo caso, a partir de las referencias teóricas y estudios realizados, el historial de desempeño de una organización hace una diferencia en el impacto de la rotación en su éxito o fracaso posterior; por lo que, este contexto organizativo se debe tener en cuenta cuando los cambios políticos se deben a temas clientelares o estratégico políticos, que nada tienen que ver con la confianza política cuando esta es complementada por la lógica de línea de gobierno y la confianza en que la gestión se realice en el marco de la respuesta a las promesas de gobierno o mejor aún de política pública.

Asimismo, la estabilidad del equipo superior puede ser una fuente de buen desempeño organizativo acorde a lo indicado por Boyne et al (2011), al menos hasta que llegue al punto intermedio del ciclo de vida del directivo según el esquema planteado para el análisis, que para el caso de estudio sería en un periodo mínimo de 3,5 años, lo que sería cercano con el periodo de gobierno. Sin embargo, el resultado del estudio evidencia a través de los datos obtenidos que, contrastando los resultados de permanencia de los directivos públicos con el esquema de ciclo de vida, únicamente la cartera de finanzas estaría dentro de la etapa 3 y próxima a la fase de mejor desempeño, teniendo en cuenta además que se trata de una relación promedio.

\section{Conclusiones}

El sistema político ecuatoriano tiene un régimen presidencialista, en el que el control sobre la administración pública se ejerce a través de la amplia capacidad presidencial de nominación y desvinculación política, del que resultan altos niveles de rotación y cortos tiempos de permanencia en el cargo de los directivos públicos. Estos indicadores contrastados con el ciclo de vida directivo adaptado al sector público por Ysa y Salvador (2015) no aseguran que la gestión llegue a la etapa de convergencia 
y de máximo rendimiento en la cúspide de la U invertida.

No obstante, se resalta la limitación derivada de la utilización de un esquema de ciclo de vida referencial, que, si bien de manera general resulta aplicable para la naturaleza de instituciones analizadas, no considera aspectos contextuales de régimen político y normativos de la gestión pública; por lo que, futuras investigaciones podrán analizar $\mathrm{y}$ delimitar ajustes al esquema, en cuanto a la duración de sus fases y sus proposiciones, teniendo en consideración las especificidades de la realidad ecuatoriana.

Mientras tanto, partiendo del esquema utilizado, si la mayor permanencia en el estudio llega a 2,6 años, significa que en términos generales únicamente en el caso del Ministerio de Finanzas se habría superado la etapa de respuesta al mandato, y la de experimentación, entrando a la etapa 3 de selección de una narrativa, es decir apalancando los nuevos paradigmas como resultado del ensayo y rectificación, un alto conocimiento de las tareas, $\mathrm{y}$, el poder desarrollado sobre la base de los resultados obtenidos de la respuesta al mandato. No obstante, la estabilidad en este ministerio sectorial responde principalmente a la permanencia de subsecretarios, es decir, el alto conocimiento de las tareas y ejercicio directivo se concentraría en el nivel político operativo de alto nivel, quedando para futuras investigaciones verificar si esta permanencia se mantiene luego del cambio de políticas que obliga al cambio de paradigmas.
Se argumenta, sobre la base de los resultados discutidos, que la permanencia de los altos directivos públicos no está aislada del cambio político o de figuras de una misma línea política, luego de un proceso electoral, y que la designación de nuevos altos funcionarios es una de las formas en que los nuevos gobernantes utilizan para afirmar sus preferencias o confianza. Queda pendiente, para próximos estudios, indagar respecto a la efectividad de la utilización de la rotación de personal como una herramienta según los resultados de desempeño de la administración previa.

En resumen, la evidencia sugiere que los niveles altos de rotación de los equipos directivos públicos sí comportarían problemas para efectivizar una dirección profesional; $\mathrm{y}$, aunque la rotación como herramienta presidencial, para designación y desvinculación de autoridades basadas en la confianza política, tiene una utilidad política y electoral, cuando los niveles de rotación son elevados, como en el caso de Ecuador, esta herramienta disminuye su utilidad estratégica.

\section{Referencias}

Alcántara, M. (2013). De políticos y política: profesionalización y calidad en el ejercicio público. Perfiles latinoamericanos, 21(41), 19-44.

Avendaño, O. y Dávila, M. (2012). Rotación ministerial y estabilidad coalicional en Chile, 1990-2010 Política/ Revista de Ciencia Política, 50(2), 87-108. Recuperado de: http://www.redalyc.org/articulo.oa?id=64 524974004 
Asamblea Nacional (2010). Ley Orgánica de

Servicio Público. Registro Oficial Suplemento 294 de octubre de 2010. Ecuador

Basabe-Serrano, S., Polga-Hecimovich, J. y Mejía, A. (2017). Unilateral no matter what: portfolio allocation in Ecuador (1979-2015). En M. Camerlo y C. Martínez-Gallardo (eds). Government formation and minister turnover in presidential cabinets. Comparative analysis in the Americas (pp. 182-206). Routledge/Taylor \& Francis Group.

Boyne, G., John, P., James, O. y Petrovsky, N. (2011). Top Management Turnover and Organizational performance: A test of a contingency model. Public Administration Review, 71(4), 572-581. Recuperado de:

http://www.jstor.org/stable/23017467,

Boyne, G., James, O., John, P. y Petrovsky, N. (2010 a). Does political change affect senior management turnover? An empirical analysis of top-tier local authorities in England. Public Administration, 88, 136-153. doi: 10.1111/j.1467-9299.2009.01751.x

Boyne, G., James, O., John, P., y Petrovsky, N. $(2010$ b). Does public service performance affect top management turnover?. Journal of Public Administration Research and Theory, 20 (2), 261-279. doi:10.1093/jopart/muq024

Camerlo, M. y Martínez-Gallardo, C (eds). (2018). Government formation and minister turnover in presidential cabinets. Comparative analysis in the Americas. New York: Routledge.

Camerlo, M. y Pérez-Liñán, A. (2015). The politics of minister retention in presidential systems: Technocrats, partisans and government approval. Comparative Politics, 47(3), 315-333. Recuperado de: http://www.jstor.org/stable/43664149
CEGE (2017). Centro de Gestión Gubernamental. Ecuador: Presidencia de la República. Recuperado de http://www.cege.gob.ec

Escobar-Lemmon, M. y Taylor-Robinson (2010). Coming or Going: How Background Affects Duration in Five Presidential Systems. En Annual Conference of the American Political Science Association, Washington, September 2-5.

Escobar-Lemmon, M. y Taylor-Robinson (2009). Getting to the Top: Career Paths of Women in Latin American Cabinets. Political Research Quarterly, 62(4), 685-699. Recuperado de: http://www.jstor.org/stable/25594440

Franco Mayorga, F. (2014). ¿Cómo mejorar la efectividad ministerial?. Revista del CLAD Reforma y Democracia, 59(2014), 55-82.

Franz, P. y Codato, A. (2018). Estabilidad e inestabilidad ministerial en el presidencialismo brasileño. En A. Codato y F. Espinoza (compiladores), Élites en las américas: diferentes perspectivas (pp.319-345). Curitiba: UFPR.

Freidenberg, F. y Pachano, S. (2016). El Sistema político ecuatoriano. Flacso, Ecuador.

Hambrick, D. y Fukutomi, G (1991). The seasons of a CEO's tenure. Academy of Management Review. 16 (4), 719-742.

González-Bustamante, B. y Olivares, A. (2015). Rotación de subsecretarios en Chile: una exploración de la segunda línea gubernamental (1990-2014). Revista de Gestión Pública, IV (2), 150-191.

González-Bustamante, B. y Olivares, A. (2016). Cambios de gabinete y supervivencia de los ministros en Chile durante los gobiernos de la Concertación (1990-2010). Colombia Internacional, 87, 81-108.doi: 10.7440/colombiaint87.2016.04 
Hernández Sampieri, R., Fernández, C., y Baptista, P. (2014). Metodología de la investigación. Sexta Edición. México: Editorial Mc Graw Hill.

Hur, Y. (2013). Turnover, voluntary turnover, and organizational performance: Evidence from municipal police departments. Public Administration Quarterly, 37(1), 3-35. Recuperado de: http://www.jstor.org/stable/24371987

Krook, M. y O Brien, D. (2012). All the President's Men? The Appointment of Female Cabinet Ministers Worldwide. The Journal of Politics, 74 (3), 840-855.

Lee, S. (2017). Employee turnover and organizational performance in US Federal Agencies. The American Review of Public Administration, 1-13. doi: $10.1177 / 0275074017715322$.

Longo, F. (2009). Introducción en Longo, Francisco e Ysa, Tamiko(eds), Los directivos públicos ante los retos de la gobernanza contemporánea en los escenarios de la gestión pública del siglo XXI, 15-36.

MDN, MEF, MINEDUC, MIES, MDI, MSP, Cancillería, MEER (2018). Portales Institucionales. LOTAIP.

Martínez-Gallardo, C. (2011). Designing cabinets: presidential politics and cabinet instability in Latin America. University of Notre Dame, Kellogg Institute Working Paper (375).

Martínez-Gallardo, C. (2012). Out of the cabinet: What drives defections from the government in presidential systems?. Comparative Political Studies, 45(1), 62-90. doi: 10.1177/0010414011421306

Martínez-Gallardo, C. (2014). Designing Cabinets: Presidential Politics and Ministerial Instability. Journal of Politics in Latin America, 6 (2), 3-38.
Ministerio de Finanzas (2017). Boletín de Ejecución Presupuestaria Octubre - Diciembre 2016. Boletín Trimestral No. 24. Subsecretaría de Presupuesto. Dirección Nacional de consistencia presupuestaria.

Ministerio de Finanzas (2017). Informe de ejecución presupuesto general del Estado. Enerodiciembre de 2016. Diciembre de 2016.

Ministerio del Trabajo (2017). Información de distributivos Institucionales para la Función Ejecutiva 2012-2016. Quito: Ecuador.

Park, T. Y., y Shaw, J. D. (2013). Turnover rates and organizational performance: A meta-analysis. Journal of applied psychology, 98(2), 268. doi: 10.1037/a0030723

PDBA (Political Database of the Americas) (2011). República del Ecuador. Gabinete. http://pdba.georgetown.edu/executive/ec uador/cabinet.html

Polga-Hecimovich, J. (2013). Ecuador: Estabilidad institucional y la consolidación de poder de Rafael Correa. Revista de Ciencia Política. 33(1), 135-160.

Polga-Hecimovich, J., Basabe. S. y Mejía, A. 2012. Shu ing the Deck: Cabinet Volatility under (Near-) Majority Government in Ecuador. Base de datos preparada para la reunión anual de la Latin American Studies Association (LASA). San Francisco, 23-26 de mayo.

Ramió, C y Alsina, V. (2016). Relación entre directivos políticos y directivos profesionales. Su impacto en términos de rendimiento institucional. $L a$ Administración Pública en el Siglo XXI, ICE, Julio -Agosto 2016, (891).

Ryu, S., y Lee, Y. J. (2013). Examining the role of management in turnover: A contingency approach. Public Performance \& Management Review, 37(1), 134-153. doi: 
10.2753/PMR1530-9576370106

Secretaría Nacional de Administración Pública, SENPLADES (2013). Plan Nacional de Desarrollo. Plan Nacional para el Buen Vivir. Quito. Ecuador.

Wulf, T., Stubner, S., Miksche, J. y Roleder, K. (2010) Performance over the CEP Lifecycle. A differentiated analysis of short and long tenured CEOs. Working Paper Char of Strategic Management and Organization. HHL. Leipzig Graduate School of Management.

Ysa, T. y Salvador, S. (2015). Liderazgo y dirección pública: el ciclo de vida del cargo directivo. Revista del CLAD Reforma y Democracia, 62, 39-76. 
